\title{
Le débat comme espace interlocutif d'identification des textes et des personnes
}

\section{Marie-Louise Martinez}

\section{(2) OpenEdition \\ Journals}

Édition électronique

URL : http://journals.openedition.org/trema/766

DOI : 10.4000/trema.766

ISSN : 2107-0997

Éditeur

Faculté d'Éducation de l'université de Montpellier

Édition imprimée

Date de publication : 1 octobre 2005

Pagination : 77 - 98

ISSN : 1167-315X

Référence électronique

Marie-Louise Martinez, « Le débat comme espace interlocutif d'identification des textes et des personnes », Tréma [En ligne], 24 | 2005, mis en ligne le 04 octobre 2010, consulté le 19 avril 2019. URL : http://journals.openedition.org/trema/766 ; DOI : 10.4000/trema.766

Ce document a été généré automatiquement le 19 avril 2019

Trema 


\title{
Le débat comme espace interlocutif d'identification des textes et des
} personnes

\author{
Marie-Louise Martinez
}

1 La démocratisation de la connaissance a buté de tous temps non seulement sur la différenciation ségrégative et inégalitaire des élèves mais aussi sur l'hétérogénéité cloisonnée des savoirs disciplinaires. Aujourd'hui cependant, à l'ère de la globalisation post - moderne de l'information, alors qu'inégalité et ségrégation n'ont pas disparu, un autre obstacle tout aussi redoutable mais malheureusement beaucoup moins perçu menace la démocratisation: celui de l'indifférenciation des personnes autant que des textes.

2 Difficilement perceptible par les théories disponibles, celle - ci se manifeste à l'école par la massification des élèves comme individus anomiques ${ }^{1}$ et par la confusion des discours et des savoirs avec contamination réciproque des types de textes, discours et genres de la culture, dans un magma incertain et uniformisé peu structurant pour la pensée. A l'école, les élèves qui ne différencient pas les savoirs et les disciplines avec leurs usages spécifiques du langage confondent tout et chaque genre scolaire non spécifié devient obstacle épistémologique pour les autres. Ils ne peuvent diversifier les postures discursives, les particularités notionnelles et disciplinaires, les types de questionnements, ils errent dans un sabir babélien qu'ils finissent par rejeter (intello !) sans parvenir à se reconnaître et se situer dans des «communautés discursives» $(\mathrm{J}-\mathrm{P}$. BERNIE²). Cette indifférenciation discursive prélude à l'illettrisme (Nicole BIAGIOLI ${ }^{3}$ ) qui est autant privation de savoirs langagiers et méthodologiques appropriés, que malaise subjectif et identitaire (mauvaise estime de soi).

3 La lecture des instructions et programmes officiels de l'Education Nationale de 2002 montre que l'importance de la différenciation des savoirs et des domaines disciplinaires commence à être vue mais qu'elle reste insuffisamment comprise dans ses enjeux et développée dans les pratiques. L'affirmation simultanée d'une double exigence de 
différenciation des champs disciplinaires des savoirs et d'une intégration interface, transversale interdisciplinaire ne manque pas de pertinence: "L'organisation progressive des enseignements en champs disciplinaires ne signifie pas, pour autant, que l'intégration des différents apprentissages de l'école primaire doive s'effacer. L'enseignant met à profit sa polyvalence pour multiplier les liaisons et les renvois d'un domaine à l'autre (...) La maîtrise du langage et de la langue française est en effet, inséparable de l'acquisition des multiples facettes d'une culture : littéraires, historiques, et géographiques, scientifiques et techniques, corporelles et artistiques. Il n'y a pas opposition entre les objectifs fondamentaux de l'école - parler, lire, écrire, compter - et des savoirs solides et différenciés $»^{4}$. Mais tant qu'elle reste allusive et incitative, elle manque l'explication des enjeux et l'opérationnalisation méthodologique qui seules peuvent transformer les pratiques.

4 La recherche en didactique et en formation, dans son approche anthropologique des savoirs disciplinaires peut prendre en charge ce défi d'une entrée transversale et comparative dans les domaines textuels des savoirs, comme d'une différenciation interdisciplinaire, inter didactique et intersubjective dans la classe. Enjeu d'autant plus important que c'est l'indifférenciation des savoirs et des personnes (non vues par les théories actuelles) qui accroît le retour d'une fabrication de la différenciation ségrégative du social à laquelle les différents observateurs de l'école sont en train d'assister aujourd'hui en toute impuissance.

5 Le débat à l'école, omniprésent dans les programmes de 2002, n'échappe pas à ces risques d'indifférenciation, il y est au contraire particulièrement vulnérable. La discussion à l'école ${ }^{5}$ est vraisemblablement l'anti - dote d'une parole magistrale, dogmatique qui cloisonne et morcelle disciplines et activités sans lien entre elles, mais sans exigence, elle peut déboucher sur la dérive inverse : propos flous et informes du café du commerce, rhétorique verbeuse qui confond les discours disciplinaires, noie la spécificité des textes et ignore la diversité des postures cognitivo - discursives.

6 Le débat à l'école, en tant que genre transversal (heure de vie de classe, débat démocratique, débat contradictoire en éducation civique, débat à visée philosophique, débat d'interprétation littéraire, débat scientifique, etc.) doit donc développer les caractéristiques communes d'un espace interlocutif de l'émergence de l'élève comme sujet singulier, réflexif et solidaire, mais chaque fois au cœur d'un savoir disciplinaire différencié avec ses visées et ses contraintes textuelles spécifiques. La prise en considération précise des particularités discursives, disciplinaires et génériques, est essentielle pour permettre l'émergence de l'élève comme personne au sein de communautés discursives ${ }^{6}$. Se servir d'un texte littéraire comme simple prétexte à une banale leçon de langage ou même à un débat contradictoire rationnel, en ignorant ses particularités de texte littéraire, serait au pire une violence de l'interprétation, un désaveu de sa spécificité discursive, au mieux un appauvrissant écrasement des genres socio discursifs de la culture.

7 Je traiterai donc ici non de l'archi - genre scolaire du débat, dans sa généralité mais d'un genre disciplinaire précis : le débat d'interprétation littéraire. Celui - ci, bien décrit par les nouveaux programmes du primaire, grâce aux propriétés du texte littéraire connues et reconnues comme telles, conduit par un enseignant formé sur la question des textes, favorise l'émergence de l'élève comme personne dans la communauté discursive d'une classe avertie sur la spécificité des textes de fiction littéraire. La recherche en cours 
d'expérimentation ${ }^{7}$ sur cette hypothèse n'autorise pas encore la vérification mais elle permet l'argumentation et la précision à la lueur d'un travail préalable dans la classe.

Je prendrai ici mes exemples dans un travail en classe mené à la croisée de deux collectifs d'étude : le GER ${ }^{8}$ « l'oral réflexif à l'école » et un groupe de réflexion d'enseignants 9 sur le débat d'interprétation littéraire. J'illustrerai plus particulièrement mon propos à partir de corpus de séances de débat d'interprétation littéraire autour de l'album Yacouba ${ }^{10}$ dans la classe ${ }^{11}$ de CM 1 de Mme Sophie ROBLIN ${ }^{12}$.

Dans un premier temps, je considèrerai l'enjeu et les modalités de la prise en compte de la spécificité discursive du littéraire ; dans un second temps, plus brièvement je ferai le lien avec la co - différenciation singulière et réflexive des élèves comme personnes au cœur d'une communauté interprétative dans la classe.

\section{Permettre l'identification des particularités du texte littéraire ${ }^{13}$}

10 Le texte littéraire par rapport à d'autres a des particularités qui ont été depuis longtemps soulignées. Malheureusement une théorie générale de la textualité n'existe pas vraiment, et celle qui se développe (notamment chez Francis JACQUES ${ }^{14}$ philosophe français du langage) à la croisée de la théorie des formes symboliques d'Ernst CASSIRER, de la text linguistic allemande et de la philosophie pragmatique du langage est encore très peu vulgarisée et transposée sur le plan didactique.

11 Comprendre un texte - tout texte, c'est saisir la particularité de son mode d'interrogation ou de questionnement et élucider progressivement le sens qu'il construit à la croisée des trois dimensions de la signifiance ${ }^{15}$ : la référence, la combinaison des matériaux signifiants et la communicabilité. Telles sont les catégories communes de la textualité, à l'intérieur desquelles chaque grande textualité (littéraire, philosophique, scientifique, médiatique, juridique, religieuse, etc.) ou domaine disciplinaire va décliner ses spécificités.

12 La sensibilisation des enseignants à cette question de la textualité, dans ses caractéristiques communes et différentielles est inexistante (si ce n'est à travers la question, la plupart du temps très ambiguë et peu claire épistémologiquement et méthodiquement $\mathrm{du}$ français transversal). Pourtant l'enjeu en est majeur, particulièrement pour des enseignants du premier degré qui en raison de leur polyvalence ne sont pas les spécialistes d'un type de textualité disciplinaire et de sa didactique. Il est urgent de bien saisir à la fois les catégories communes des différents types de textualités disciplinaires et ce qui les distingue, afin de permettre aux élèves de bien différencier comparativement les textes et domaines du savoir.

Dans la classe de Mme ROBLIN les débats d'interprétation littéraires (enregistrés en 2003) sont familiers aux élèves, la démarche de l'enseignante, délibérément peu systématique, reste tâtonnante et exploratoire quoique empreinte d'une grande expertise. La discussion est riche et productive, malgré les obstacles rencontrés par les élèves et les approximations. Le bénéfice pour l'identification spécifique du littéraire, et l'identification subjective de chaque élève est évident.. Je crois cependant qu'une sensibilisation préalable sur les textes et sur l'approche spécifique et différentielle (interdidactique) aurait encore augmenté l'efficience. 
14 Je présenterai l'analyse selon les 4 catégories fondamentales de l'approche textuelle, en insistant chaque fois sur la spécificité du littéraire. On verra que même si celles - ci n'ont pas été utilisées explicitement avec les élèves, elles ont été prises en compte pour saisir la particularité du texte littéraire.

\section{I.1. Le questionnement littéraire : une énigme ouverte à une pluralité d'interprétations :}

Bé moi j'ai pas trop bien compris l'histoire, dit Manon (I - 2) ${ }^{16}$ après la lecture du texte, Julien (I 13), à son tour avoue : Bé moi j'l'ai pas très bien compris pourquoi il a pas tué ++, et un élève (I - 23) résume la situation : J'ai presque pas très bien compris.

Jusque vers à la fin du débat pour Manon (I - 369), qui pourtant a découvert bien des choses, le clair obscur énigmatique demeure :j'ai pas très très bien compris.

Manon et Julien expriment pourtant par leur perplexité même qu'ils ont compris l'essentiel: le texte littéraire est toujours une énigme qui excède la compréhension immédiate. Le sens littéraire se fait progressivement jour à travers un cheminement dialogique où le lecteur est convié par l'auteur à construire des significations dans l'inter - prétation ${ }^{17}$. Le propre du texte littéraire, à la différence du médiatique, du scientifique ou du philosophique, est de permettre cette posture interrogative où le sens s'élucide progressivement à travers la pluralité maintenue ouverte et non univoque (presque pas très bien compris!) des significations possibles. Seul le littéraire (particulièrement fiction et poésie) ouvre à cette polysémie où le sens cependant ne cesse jamais d'être très contraint par le texte (tout et n'importe quoi ne peut pas être dit à partir d'un texte littéraire qui demande une lecture attentive et rigoureuse des indices). Significations très contraintes et cependant plurielles: sens multiples que la rencontre avec une époque et un lecteur dans sa singularité saura lire (élire) avec bonheur. Paradoxes, métaphores, ellipses, etc. maintiennent une sorte d'indécidable qui peut, de prime abord, donner le vertige à un jeune lecteur. Peut - être faut - il une certaine maturité (qu'il contribue sans doute à produire) pour accepter qu'on ne saurait dire ni comprendre le dernier mot d'un texte littéraire toujours ouvert.

16 Au long des débats, avec souplesse, sans ordre préétabli, mais selon un rituel structurant qu'ils reconnaissent, les élèves de cette classe sont conduits à interroger le texte.

\subsection{La référence : de quoi parle le texte?}

Les approches structuralistes ont trop longtemps effacé la dimension référentielle du texte littéraire, seule la signification immanente (matériaux signifiants) leur paraissait importante. Or, d'autres approches ont mis en lumière que la littérature n'était pas un pur jeu de significations internes et structurales. Elle renvoie au contraire, avec force, à des réalités extérieures.

L'approche psychologique montre que la littérature évoque des univers fantasmatiques privés et cependant partageables grâce à leur symbolisation. Pour l'approche sociologique, l'aptitude du texte littéraire est d'exprimer, au plus haut degré, les « visions du monde » des groupes sociaux. Pour l'approche anthropologique ${ }^{18}$, enfin, la littérature peut selon sa qualité refléter ou dévoiler le désir et les processus de la violence et du 
sacré, de l'homme en relation. Telle l'énigme du sphinx, la littérature dirait une vérité essentielle sur l'humaine condition avec encore plus d'acuité que d'autres textes (philosophie, sciences, etc.).

- Comprendre la référence aux objets du monde, pour les élèves, c'est d'abord entendre le lexique et les notions. Les termes dans Yacouba sont très simples sauf rival, sacré et banni qui sont écran, car ils renvoient à des réalités notionnelles sociales ou anthropologiques. Celles - ci sont perçues progressivement par les élèves, d'abord tautologiquement, puis plus précisément définies.

Johanna (I - 11) « Bé comme l'histoire enfin ya une sorte de village voilà y'a des tribus qui chassent avec des lances ça me fait penser à ça voilà »

Maîtresse (I - 18I) Au début y'avait écrit " c'est une grande journée »

Rufin (I - 182) C'est sacré

(...)

Maîtresse (1 - 4) «Peut - être y a un mot que tu n'as pas bien compris quand je l'ai lu euh +++ là c'est le lion il dit "j'ai combattu toute la nuit contre un rival féroce «qu'est - ce que tu as compris toi à ce moment là ?»

Thomas (I - 44) «A mon avis c'était pas Yacouba parce que ça devait être un autre animal» Jérémy (II - 81) «Euh rival ça doit être un animaux un animal »

Maîtresse (II - 86) «Un autre lion c'est sans doute un autre lion un rival c'est quelqu'un qui est qui se situe sur le même plan et qui veut prendre la place de l'autre en général donc il a combattu un autre lion "

(...)

Thomas (I - 306) « Banni si dans ce livre banni il veut dire qu'il sera pas gardien »

Johanna $(\mathrm{I}-310)$ «banni de tes frères qui sera rejeté enfin voilà »

- C'est comprendre aussi l'univers social et culturel de référence : la tribu d'Afrique et le rite d'initiation s'élucident dans la coopération explicative des élèves :

Manon (I - 193) «Ils deviennent guerriers en battant des lions»

Ruffin (1 - 195) «ça prouve qu'ils sont forts et qu'ils ont du courage »

Thomas (I - 196) «Parce que s'ils avaient pas à combattre on saurait pas s'ils sont courageux et forts. »

- C'est comprendre les hiérarchies sociales du monde évoqué dans la fiction : devenir un guerrier dans cette société est davantage valorisé que devenir un guardien de bétail. Mais cette compréhension est toujours relative au monde vécu par le lecteur. Pour nos jeunes lecteurs camarguais qui vivent dans les mas de manades et dans l'admiration des guardians et des farouches taureaux, la suprématie du guerrier n'est pas évidente :

«Et bé ils deviennent guardiens ... guerriers » Pour Thomas (I - 139 - 141) le gardien (le guardian ?) n'a rien à envier au guerrier pour le courage. Et Ruffin renchérit (I - 237) : « et là aussi il est comme un guerrier ; le bétail c'est des gros taureaux et d'habitude y'a le lion qui vient attaquer» 

coopération cognitive hypothético - déductive très serrée se noue entre Manon, Johanna et Rufus pour déterminer à quoi servent les guerriers dans cette tribu :

de (I’45 à I - 58), alors si ... et si ne pas ... Alors donc (...) «donc à défendre sa tribu contre d'autres guerriers des autres villages $»$.

- C'est comprendre les valeurs morales, par - delà les valeurs sociales : les valeurs en jeu dans ce texte sont progressivement comprises par les enfants dans la discussion. Manon et Johanna sentent bien qu'il y a pour Yacouba le franchissement d'un seuil anthropologique ; si le courage de tuer le lion est grand, celui de l'épargner est supérieur :

Manon (I - 156) « il va être plus que les autres, pas guerrier mais encore plus que les autres»; Johanna (158) «plus élevé que les autres ». Un élève (I - 160) «plus courageux, plus combattant »; Johanna (I - 161) «plus intelligent ...»

Pour Thomas il est clair que Yacouba renonce à un rôle social prestigieux, au partage des valeurs communautaires et même à un désir propre au nom d'un bien supérieur (même naïvement formulé comme amour des animaux).

Thomas (I - 27) « il a pas tué parce qu'il préfère pas tuer; (I - 84) il a abandonné sa tribu et son espoir de devenir guerrier, (I - 91) parce qu'il aime les animaux»

Pour Jérémy, dans un autre groupe, Yacouba accède à une réflexion et à la conscience morale par sympathie (souffrir pour l'autre) :

Jérémy (II - 575) : «Et après et après quand il a quand il a réfléchi il se dit euh non euh non je dois pas le tuer» (II - 583) «c'est un peu comme s'il avait de la peine pour le lion»

Et Mélodie élucidera avec finesse le sens existentiel altruiste trouvé par le personnage : sacrifier un désir propre et renoncer à un bien personnel pour le bien supérieur d'un autre :

Mélodie (II - 443) « il a prouvé qu'il avait du cœur il a préféré laisser la vie sauve au lion que de devenir guerrier il a laissé en quelque sorte tomber son rêve»

L'usage approximatif du lexique axiologique, donne des naïvetés qui peuvent faire sourire l'adulte : (être lâche n'est pas forcément négatif pour Johanna : c'est savoir lâcher le lion ! ). Quoiqu'il en soit, cette compréhension axiologique (comparaison hiérarchisée entre des valeurs) ici est prise dans la singularité (du lecteur comme du personnage), elle n'est pas abstraite et générale comme il serait de rigueur dans la discussion à visée philosophique, 
elle est saturée du concret d'une intrigue particulière avec les motivations propres et subtiles d'un personnage pris dans les rets de relations singulières.

Mais même si les obstacles demeurent pour certains dans la compréhension de la référence du texte (l'allusion elliptique du littéraire n'explique pas assez selon eux), ils ont appris que cela demande une relecture plus fine des indices du texte et de l'organisation interne des matériaux langagiers utilisés. Leur pratique spécifique du texte littéraire les a habitués à cette relecture avec recherche d'indices susceptibles de vérifier ou non une interprétation.

Enfin, les enfants ne se contentent pas d'évoquer les références sémantiques du texte, ils le lisent dans sa forme (la morphosyntaxe) et les matériaux propres à la textualité littéraire. Les différentes dimensions de la signifiance sont interactives et imbriquées.

\section{I.3. L'organisation et la structuration des matériaux signifiants : comment et avec quels moyens langagiers?}

Il s'agit ici de repérer les particularités du texte littéraire dans l'articulation et la combinaison des signes entre eux, constituées par les procédés littéraires.

Dans cette classe, les élèves sensibilisés à la littérature, repèrent assez vite les procédés littéraires, sans pouvoir pour autant les décrire précisément. Leur verbalisation très approximative n'en est pas moins évocatrice :

Manon (I - 25) «C'est quand le moment qu'il se bat contre le lion, où il y avait pas d'image, où y avait les décors euh les + on dirait comme une sorte de récit qui disait et + moi j'ai pas très bien compris là»

Johanna (I - 26) «'est quand ... y'avait que les décors ...c'est le lion qui parlait ... »

Dans Yacouba, un seuil anthropogénique va être franchi dans l'identification : celui qui va du meurtre sacrificiel de l'animal totémique, lors du rituel initiatique, habituel pour consacrer une identité de guerrier programmée et valorisée par la tradition clanique (identité meurtrière), vers une autre initiation plus intime où le personnage va accéder à une autre identité : celle du sujet de la conscience. Contrairement à l'identité tribale traditionnelle (guerrier) fondée sur le sacrifice d'un autre (ici le lion), l'identité du sujet (accès à la Loi morale) au contraire est fondée sur le respect de la vie de l'autre. C'est bien parce qu'il renoncera à accomplir le geste tribal qui lui donnerait la position sociale désirée que Yacouba inaugurera l'invention d'une sorte de rite nouveau (le débat intérieur de la conscience réflexive) qui lui permettra d'accéder à une identité d'un type nouveau. L'une s'inscrit dans une culture particulière l'autre dans une intériorité à la fois plus singulière et universelle. Ce passage anthropologique, franchi dans le temps historique par le passage d'un type de société à l'autre, est indiqué et signifié dans l'ouvrage de Thierry DEDIEU en raccourci dans la conscience du personnage par un procédé simple (véritable trouvaille littéraire) qui atteint ici sa force première : le passage du récit au discours. 

violence est désigné par la troisième personne dans le récit. Le récit, même de type romanesque, est encore proche du mythique comme fondation violente de la communauté tribale. Le «l» est non personne dans le récit, nous rappelle BENVENISTE ${ }^{19}$. Son étude sur la particularité du statut du tiers personnel vient étayer le postulat de l'éviction du tiers dans l'anthropogenèse violente et le rôle fondateur de l'absent. Mais dans le discours, forme interlocutive du langage où les personnages en présence s'interpellent (je - tu), le il du récit (le lion) dit je et s'adresse directement au héros qu'il appelle (le tu devra répondre à son tour en je, à la première personne) à la conscience à la responsabilité (qualité acquise justement par celui qui accepte de répondre à l'appel) du sujet parlant et agissant. l'iconographie figurative s'interrompt pour laisser place à une sorte de cadre graphique plus neutre. Cette frise rompt avec la représentation de l'image, elle exhibe et magnifie l'importance du langage verbal écrit dans sa force de loi symbolique. Ici on est sorti de la référence figurative (même stylisée), les mots, marqués par l'arbitraire des signes, ne reposent plus dans leur portée significative que sur la convention et l'accord des hommes entre eux. Fragile accord qui fait la Loi avec ses inter - dits conventionnels. Cette irruption d'une parole mise en valeur (encadrée) évoque l'écrit fondateur mosaïque des tables de la Loi. Cette solennité n'échappe pas à nos jeunes lecteurs : la plongée dans les yeux du lion et la lecture du discours écrit encadré sont une sorte de " commandement» qui permet l'entrée dans la Loi (avènement du sujet réflexif selon $\mathrm{KANT}^{20}$ ), comme l'entrée dans le symbolique. Il vient illustrer l'injonction nouvelle découverte par le personnage de Yacouba : tu ne tueras point. Désormais il peut s'affranchir des injonctions de la tribu (hétéronomie), il obéit à l'interdit fondateur du sujet moral (autonomie). Dans la textualité philosophique, le sujet fut formulé par KANT au dix - huitième siècle des Lumières comme rationalisme universel, il restait à le redécouvrir dans le visage de l'autre singulier. Ce sera fait assez tardivement par Emmanuel LEVINAS ${ }^{21}$, ou Francis JACQUES $^{22}$ philosophes de la subjectivité de l'Autre (comme toi ou comme lui), dans la singularité vulnérable de son visage.

Comprendre ces références anthropologiques à travers une lecture des matériaux langagiers est difficile mais les élèves ici ont appris à scruter et à interroger en communauté interprétative soucieuse des contraintes du texte ces procédés de rupture énonciative, ce décalage entre le texte et l'iconographie non illustrative.

C'est bien ce que Cindy nous dit, dans une coopération explicative avec Alexandre, grâce au truchement de l'enseignante :

Alexandre (II - 9) «les yeux du lion»

Maîtresse (II - 10) «Ah comment tu vois les yeux du lion»

Alexandre (II - 11) «Bein comme ils disent on + on peut lire dedans»

Maîtresse (II - 12) « est - ce que Cindy tu imagines que ça peut être les yeux du lion»

Cindy ( II - 13) « et Yacouba il voit ce c'qui y marqué dedans et il veut pas le tuer» 

même souvent le verbal. Elles disent une vérité de la réalité mieux que les mots : la fête est triste, les visages sont fermés et les couteaux se brandissent car le sacré, ici, est fondé sur le rite violent et sur la mort. Les images, loin de refléter le réel factuel, évoquent le monde fantasmatique, elles disent, par exemple, à quels désirs ancestraux de meurtre Yacouba devra résister pour épargner le lion. Cette articulation contrastée des matériaux signifiants du texte (système verbal et iconographique) fait partie des codes du genre de l'album de jeunesse. y était - il personnellement impliqué ? Cindy, Alexandre, Mélodie, Adrien, et les autres vont enquêter et scruter patiemment le texte (de II - 520 à II - 584), avec la maîtresse, ils font des hypothèses et les vérifient par une relecture attentive et croisée de l'écrit et des images. 
- Yacouba lui lui il voulait pas être guerrier donc il a pas tué le lion (...)

- Non parce que + + bé Yacouba on voit on enfin j'sais pas si c'est Yacouba parce qu'au bout d'un moment vers une page on voit qu'y'a quelqu'un qui se lance avec un couteau j'sais pas si c'est Yacouba (..)

(...)

- Là y'a un couteau

(...)

- Dans le village on désigne

- On choisit ceux qui vont qu'est - ce qu'il fait là

(...)

- XXX (relecture marmonnée du texte)

- Là là il a là j'pense il a il est en train de regarder euh

- Ils ont mis «puis soudain»

(...)

- Ah attendez on y arrive là Yacouba + il cherche

(...)

- Il observe " puis soudain» Le lion

- Il sort les dents

- Et qu'est - ce qu'on nous dit?

- «S'armer de courage»

(...)

- Donc là c'est Yacouba!

- «S'élancer pour combattre»

(..)

- Donc peut - être qu'il veut qu'il veut le tuer quand même parce queuh un peu là

(...)

- Et après et après quand il a quand il a réfléchi quand il a réfléchi il se dit euh non euh non j'dois pas

l'tuer parce que

(...)

- Si il avait rencontré un lion qui n'était pas blessé

- Il l'aurait tué

- Là il aurait carrément - Et il s'rait devenu

- guerrier

38 Ces jeunes lecteurs ont appris à s'engager ensemble dans la recherche des indicateurs textuels où ils discernent l'implicite.

Mais comment savent - ils se situer par rapport à cette autre dimension de la signifiance la plus déterminante pour la compréhension du texte littéraire qu'est la communicabilité?

Il ne suffit pas de comprendre une histoire et une série d'idées ni même de décrypter la combinatoire des matériaux pour lire véritablement le texte littéraire, encore faut - il le saisir dans la polyphonie de son dispositif de communication. Le réseau de places énonciatives et interlocutives dans le texte littéraire est complexe et subtil : à travers la multiplicité des places énonciatives, à travers le dialogue des personnages qui nous parle, et pour nous dire quoi ? Comprendre toute la dimension interlocutive : le dialogue entre les personnages s'insère dans un autre jeu dialogique plus essentiel entre l'auteur et le lecteur. C'est là sûrement, dans ce dispositif interlocutif croisé que réside la vocation 
spécifique du texte littéraire, l'ignorer serait se priver d'une modalité essentielle de la culture et de l'esprit. Sans cet apprentissage précoce, comment déceler le réseau interlocutif de personnes réelles derrière les personnages simples porte - voix mais pourtant plus réels que de simples êtres de papier? Comment repérer la visée profonde de l'auteur comme énonciateur dans son adresse au lectorat? Comment sans cela comprendre un film, une pièce de théâtre, un roman, comment s'ouvrir aux œuvres les plus complexes et les plus significatives de la culture universelle et les relier entre elles?

41 A travers ce débat d'interprétation, les élèves ont trouvé quelques pistes pour entrer dans la communicabilité du texte littéraire. Ils ont appris à se sentir visés par une intention de communication (qu'est - ce qu'on nous dit?) à se positionner en communauté de lecteurs (nous) qu'un on (l'auteur encore indéfini) voulait toucher. Ils cherchent à cerner l'intention de l'auteur (dans l'indéterminé qui l'entoure) et sa visée sur les jeunes récepteurs culturels qu'ils sont. Ils entrent ainsi dans l'universel réseau de la culture lettrée dont ils s'estiment désormais destinataires de choix.

\section{I.4. La communicabilité et le dispositif interlocutif : qui s'adresse à qui, pourquoi, pour quoi?}

Selon le linguiste Mikhail BAKHTINE ${ }^{24}$, on peut déceler dans la textualité littéraire l'entrecroisement de deux dialogismes. Le dialogisme externe (dialogue entre auteur(s) et lecteur(s)) ou extradiégétique ${ }^{25}$ et le dialogisme interne ou intradiégétique (dialogue entre les personnages). J'ai soutenu ${ }^{26}$ que les personnages appartiennent aux deux dialogismes, non seulement le dialogisme interne de la fable dont ils sont les actants, mais le dialogisme externe car ils sont les intermédiaires : entités de parole crées par l'auteur autant que supports identificatoires du lecteur ou du spectateur. Ils constituent ainsi les noeuds du réseau, les points de capitons de ce maillage transactionnel qui articule et réticule l'actuel avec le fictionnel, le réel, l'imaginaire avec le symbolique. Dans le jeu théâtral, l'acteur incarne cette mise en chair du personnage fictionnel qu'il re - présente, dans une présence réelle ou du moins symbolique. Pour comprendre le texte littéraire, le lecteur doit se re - présenter lui - même dans les différentes instances du texte et se positionner dans les différentes postures énonciatives: se mettre à la place du personnage et de ses protagonistes, voire de ses antagonistes, se prendre tantôt pour l'auteur et tantôt pour le metteur en scène. La lecture est une véritable gymnastique de l'Autre qui passe par l'adoption de la posture de l'autre, d'autres multiples et souvent antagonistes, elle demande souplesse, ouverture à l'altérité, imagination, capacité de décentrement par rapport aux cadres étroits de nos propres représentations égocentrées. Exercice particulièrement difficile pour les jeunes enfants. Et pourtant depuis les contes de nourrice ne sont - ils pas rompus à cet exercice, ce jeu de l'autre?

Le texte littéraire est sans pareil pour ouvrir à l'altérité :

Johanna et Manon (I - 41 - 43) se projettent dans la vie psychique de Yacouba : "Même il a du faire des rêves après et c'est peut - être quand il a fait un rêve ça a du peut - être guider " ... "et ça l'a aidé à prendre sa décision » 
Dans la relation intradiégétique, ils ont su chercher les indicateurs et voir que si l'acte de tuer le lion était « désigné» et commandité par les adultes du clan, le désir propre de Yacouba y était largement investi.

Thomas, Johanna (I - 84 à I - 94) mesurent combien le « renoncement » en faveur du lion en sera plus coûteux :

«Il en rêvait dès qu'il était tout petit (...) Il a abandonné sa tri sa euh son espoir de devenir guerrier »

Adrien (I - 606) parvient à s'imaginer à la place de l'auteur :

« Moi je j'étais l'auteur et queuh que j'avais imaginé qu'il avait tué et bée je j'aurais continué l'histoire. »

D'autres comme Thomas et Rufin (I 421 - 425), en fins connaisseurs de l'institution littéraire, s'intéressent à la dimension sociale de la production de "l'écrivain », aux collections, au circuit »d'édition» et de parution :

"L'écrivain il pourrait rajouter un peu de ce qui se passe après» "Maîtresse il en a fait qu'un livre comme ça? "Ya pas de collection? Vous l'avez eu où?"

D’autres enfin, s'installent dans la posture évaluative légitime du lecteur autorisé pour émettre des avis motivés :

Alexandre (II - 11) « Moij'l'ai aimé parce qu'il tue pas le lion voilà »

Adrien (II - 1) «Euh je dis bein moi euh j'l'ai pas beaucoup aimé parce que çà + la la l'histoire ne raconte pas assez pour moi elle raconte pas assez + de choses voilà »

9 La communicabilité d'un texte s'étend souvent en réseau avec d'autres textes de la culture qu'ils évoquent explicitement ou implicitement, ce phénomène a été nommé l'intertextualité par les théoriciens de la littérature, comme GENETTE. Ce pouvoir évocateur d'une œuvre qui résonne avec d'autres œuvres pour constituer l'univers de la culture a été souligné par certains didacticiens comme DEVANNE : il donne à l'élève qui commence à s'y frayer un chemin de reconnaissance, un fort sentiment d'appartenance à la communauté lettrée. La communauté discursive littéraire.

Ici, les élèves semblent initiés à cette mise en résonance culturelle, avec liberté et pertinence, ils affirment leur compétence de lecteur et de spectateur: Manon (I - 4) pense, avec finesse, au célèbre roman jeunesse de Daniel PENNAC :

« Il m'a fait penser un peu à L'œil du loup parce que déjà à un moment donné il se regarde dans l'œil + dans le regard profond ça fait penser un peu à L'œil du loup » 

conte :

«Ça fait un peu comme Blanche Neige quand euh le chasseur il veut la tuer mais il peut pas et lui c'est euh sauf qu'il est blessé mais il peut pas la tuer parce qu'il est + »

D'autres, s'inscrivent (avec une certaine naïveté), comme membres d'une culture partagée et comparent le texte à d'autres albums ou à des émissions de télévision :

\footnotetext{
"Moi aussi ça m'a fait penser au livre de Kirikou pasque heu c'est pareil ya une tribu euh Kirikou doit chasser la reine " (...) «Sur la cinq, y'a + ya une sorte de village voilà y'a des tribus qui chassent avec des lances ça me fait penser à ça voilà »
}

Dans cette classe, on voit bien (malgré l'aperçu trop bref de deux séances) que les élèves sont en train de construire des compétences concernant l'analyse d'un texte littéraire et le genre spécifique du débat d'interprétation littéraire. Ils ont appris à questionner et à élaborer collectivement les significations dans la compréhension interprétative de la référence, des matériaux langagiers et de la communicabilité.

On peut constater combien cette pratique avertie du roman pour la jeunesse contribue à permettre l'identification des élèves comme membres à part entière d'une communauté discursive et culturelle partagée (nous). Ils peuvent se référer à un patrimoine littéraire ou culturel connu (romans pour la jeunesse, contes, émissions de télévisions), utiliser un métalangage ou plutôt un périlangage spécifique (récit, personnage, auteur, édition, collection, etc.).

Ils acquièrent estime de soi et sentiment d'une affiliation scolaire et culturelle partagée. Cette ouverture à l'autre rejaillit sur la socialisation dans la classe : quand ils discutent pour établir au plus juste les significations du texte, ils s'accordent réciproquement le droit à diverger dans la réception du texte, non seulement par la plurivocité du texte mais par un respect de la diversité des sensibilités. Cette affiliation les inscrit dans une appartenance spécifique, leur donnant la reconnaissance d'une compétence effective et d'une identité générique et scolaire, ces élèves commencent très solidement à entrer dans une approche spécifique de la littérature. Celle - ci pour eux n'est pas simple prétexte à réfléchir à une question morale, elle est un texte dont la lecture exige un certain nombre de compétences, de postures et d'interrogations pour être comprise dans ses références, ses matériaux signifiants et dans sa communicabilité.

Pour mettre cette compétence littéraire en construction à l'épreuve d'autres compétences disciplinaires, il aurait été intéressant de faire vivre aux élèves un débat à visée philosophique, un débat scientifique, un débat démocratique comme en éducation civique. C'est cela même que recherche l'approche interdidactique ${ }^{27}$ afin de mesurer la construction d'une compétence différentielle des textes et des disciplines. C'est vers cet objectif que se dirige notre recherche actuelle. Mais cette indispensable différenciation des textes, ne doit pas être déconnectée du souci essentiel pour les enseignants de différencier les élèves et de leur permettre à travers le processus d'apprentissage de s'identifier comme sujet et personne (véritable fin de l'éducation). 


\section{Permettre l'inter - identification des élèves comme sujets et personnes}

57 Il ne va pas de soi de permettre que la classe soit un espace interlocutif de l'émergence de l'élève comme sujet et comme personne. L'enjeu identitaire et éthique est cependant au coeur de nos sociétés en crise. Or les phénomènes d'identification et d'interidentification passent par les interactions langagières dans la classe, où se jouent conjointement la co construction des savoirs, la définition même de l'institution avec ses rôles réciproques et la définition des identités. Ils demandent une attention constante de l'enseignant, car s'y jouent de manière croisée les deux principales finalités de notre école républicaine (l'instruction et l'éducation) sans compter la fin anthropologique de l'éducation: l'émergence de l'enfant comme élève et comme personne. Je prétends que cette compétence transversale n'est pas seulement éducative ni même pédagogique mais qu'elle est didactique c'est - à - dire qu'elle concerne le rapport aux savoirs enseignés.

\section{1. Le sujet singulier de l'énonciation}

S'identifier comme sujets singuliers (sujets non assujettis d'un désir sinon propre ou du moins réapproprié) pour les élèves, c'est pouvoir s'investir dans une énonciation qui manifeste une certaine autonomie par rapport à la parole ou au désir de l'enseignant, mais aussi par rapport à celle des autres élèves. La théorie de l'énonciation de BENVENISTE a complètement renouvelé la compréhension linguistique de la notion de personne et a eu un grand impact sur la conception actuelle du sujet. Pour BENVENISTE le sujet est le résultat d'un acte d'énonciation (production d'énoncés) par lequel le sujet parlant assume le langage : « c'est dans et par le langage que l'homme se constitue comme sujet : parce que le langage seul fonde en réalité, dans sa réalité qui est celle de l'être, le concept d'ego $»^{28}$... C'est grâce aux embrayeurs, mots qui indiquent le temps, l'espace (ici, maintenant) mais, surtout, aux pronoms personnels de la première et de la deuxième personne, que le sujet peut assumer le langage dans le discours. Ainsi, pour le linguiste, par l'acte d'énonciation, le sujet parlant se confère à lui - même, en quelque sorte, une véritable consistance ontologique qu'il puise dans le langage. S'autoriser à parler, assumer la parole, (assumpta parabola) est l'acte même de l'audace de l'ontologisation de soi. Cette identification du sujet de l'énonciation est essentielle si l'on veut que le rapport au savoir de l'élève soit investi par quelqu'un, lui - même comme sujet.

Dans les interactions habituelles de la classe traditionnelle où les élèves répondent passivement à tour de rôle aux questions et aux injonctions de l'adulte, cet investissement énonciatif existe très souvent mais il peut difficilement se remarquer. Il apparait dans les débats, lorsque le groupe d'élèves, plus autonome gère la prise de parole à partir des règles qui ont été instituées avec l'adulte. Chacun alors selon son intérêt, va illustrer la thématique choisie, selon son inclination et son rythme propre, il va questionner le texte. C'est le cas dans une longue séquence (I 271 - 282) où Johanna, Manon, Ruffin, discutent vraiment entre eux dans une dynamique très interactive et où ils expriment leur avis avec franchise et sans complaisance pour l'avis magistral d'ailleurs non exprimé. On assiste même ici à une relative inversion des rôles, puisque c'est une élève Johanna qui demandera à l'enseignante de leur faire la lecture pour vérifier le texte : 
Johanna : Bah moi j’aurais pas tué il était à moitié mort ... Manon : Et pourquoi il était ... Ruffin : Et pourquoi tu l'aurais pas ... Manon : Et pourquoi tu dis qu'il était à moitié mort ... Johanna : Parce qu'il voulait pas tricher une sorte de euh de laisser en vie ... Ruffin : Moi je l'aurais tué si c'était si c'était si c'est vraiment mon avis d'être guerrier euh je l'aurais tué quand même... Elève ( $\mathrm{T}$ ?) : Ah ouai ... Ruffin : Bah oui si ... E : XXX ... Ruffin : Bah oui puisque ça fait puisque après il devient guerrier et il a envie de devenir guerrier et il sait que c'est comme ça qu'il deviendra guerrier ... $\mathbf{E}$ : Et oui Johanna : Bé oui et bah soit tu soit il soit il soit il lâche on pourrait revenir à la page où le lion parle Maîtresse : Oui tu veux que je relise ... Johanna : Oui les deux ... Maîtresse : comme tu peux le voir je suis blessé. J'ai combattu toute la nuit contre un rival féroce. ... E : arrête ... Maîtresse : Tu n'auras donc aucun mal à venir à bout de mes forces. Soit tu me tues sans gloire et tu passes pour un homme aux yeux de tes frères, soit tu me laisses la vie sauve et à tes propres yeux tu sors grandi mais banni tu le seras par tes pairs

BENVENISTE a mis en lumière la puissance conférée au sujet par cet acte de simple prise de parole. Cette audace ne saurait bien se comprendre et se saisir que par rapport à sa part d'ombre. Tous ceux qui jamais ne se risqueront à prendre la parole, les muets, ceux qui tremblent et bégaient, les autistes, disent au moins combien l'assomption de la parole est difficile. Pour la problématique éducative, l'assomption de la parole est un fait intersubjectif et relationnel, tout comme l'est son impossibilité. Cela ne peut se comprendre que si l'on prend toute la mesure du contexte de rivalité potentielle qui accompagne la parole, lors de son apprentissage comme lors de son difficile partage, dans les circonstances de la vie quotidienne, sociale et institutionnelle. Benveniste a entrevu la menace toujours présente de l'aliénation ${ }^{29}$.

\section{II.2. Le sujet de l'autonomie}

61 Car il ne suffit pas de dégager un espace de liberté aux élèves par rapport aux injonctions du maître pour leur permettre d'accéder à l'autonomie. Celle - ci, en effet, ne doit pas seulement être comprise comme sortie de l'hétéronomie (où l'enfant demeure sous la coupe de l'autre adulte) mais aussi de l'anomie où l'enfant oscille sans repères entre les mimétismes et les pressions des pairs.

La séquence II (2 - 10) commence par un temps de débat autogéré par les élèves Jérémy, Cindy, Mélodie, Rosine, Alexandre où Adrien tient le rôle d'animateur. Mais comme les enfants ne connaissent pas bien encore les ressources du débat, qu'ils ne se sont pas appropriées ses règles, cela tourne vite court et chacun va se calquer de manière indifférenciée et passive sur un mimétisme paresseux du propos des autres.

Adrien : Maintenant qu'est ce que c'est votre avis après avoir discuté Jérémy ... Jérémy : Euh ++ bé de + j'sais pas c'est c'est pareil c'est enfin le lion le lion d'façon il Yacouba il a du réfléchir et et lui dans sa tête il a du se dire et bé moi je vais pas le tuer sinon ça j’aurais ça ça aurait rien représenté pour moi ... Adrien : Mélodie ... Mélodie : Bé pareil ... Adrien : Cindy ... Cindy : Pareil ... Adrien : Rosine ... Rosine : ++ je sais pas ... Alexandre : Pareil ... Adrien : Bé moi c'est pareil parce qu'au début c'est c'que je voulais dire voilà. 
63 savoir qui va décider l'enseignante à mettre en place tout un dispositif d'étayage. Tour à tour elle va:

- focaliser l'attention des élèves sur les problèmes posés par le texte :

II 54 Je voudrais qu'on s'arrête sur ces deux pages là

II - 91 Et alors quel va être le problème de Yacouba?

- inciter à l'explicitation :

I - 95 Qu'est - ce qui se passe s'il lui laisse la vie sauve?

I - 97 Et pourquoi ça? Et pourquoi il faut aller combattre le lion?

- faire circuler les questions :

II - 154 Alors qu'est - ce qu'on peut répondre à la question de Cindy?

- ramener sans cesse au texte qu'elle relit ou fait relire :

II - 169 je crois qu'on peut revenir sur un grand silence accueillit Yacouba

- souligner les conflits d'interprétation :

II - 242 Alors Cindy est - ce que tu es d'accord avec Jérémy?

- protéger la parole des plus timides :

I - 400 On laisse finir Rosine, tu vas parler après

Grâce à cet étayage (BRUNER) l'enseignante travaille ici véritablement dans l'espace proximal (VYGOSTKI) de chacun, en mettant en œuvre une connaissance différenciée des élèves. Chacun peut alors investir à sa manière le savoir et se retrouver impliqué dans la relation avec les autres et avec l'ensemble. Il peut contribuer à co - construire la compréhension autant que la signification du texte, à co - construire les règles d'une communication partagée. Sortant de sa toute (im) puissance, chacun peut alors se percevoir comme 'un parmi d'autres' sujet de son désir mais aussi sujet non assujetti, convenant à la règle commune. Cela est rendu possible par le partage de la convention symbolique, pour BENVENISTE, la subjectivité considérée hors du langage n'est qu'illusion ou reflet, car, en fait, elle est (elle n'est que) : " ... l'émergence dans l'être d'une 
propriété fondamentale du langage. Est ego qui dit ego. Nous trouvons là, le fondement de la subjectivité qui se détermine par le statut linguistique de la personne $»^{30}$.

\section{Le sujet de l'interlocution comme personne}

Dès lors que l'enseignant protège la coopération et le sain conflit cognitif, l'élève n'est plus seulement le sujet de l'énonciation, il peut bénéficier pleinement de l'interlocution : celui qui se co - construit et émerge dans l'échange partagé de la parole. Le fruit de l'entrée dans l'échange langagier rituel est non seulement la pensée mais aussi l'identité, cette ontologisation de soi par l'échange intersubjectif. Toute la sphère du symbolique est fondée sur le sacrifice et le don dira BENVENISTE en évoquant les travaux de l'anthropologue Marcel MAUSS, il permet de s'engager dans l'échange et de donner pour recevoir " ... les rapports humains sont fondés sur la réciprocité des prestations privées ou publiques, dans une société où il faut s'engager pour obtenir $»^{31}$. Les catégories linguistiques sont les instances médiatrices qui donnent au sujet l'occasion de s'identifier (se rendre identique autant que se différencier). Ce processus complexe est, en effet, permis par la catégorie linguistique substitutive par excellence, celle de la personne. Catégorie universelle, elle existe dans toute langue et dans toute culture, à toute époque ${ }^{32}$.

Grâce à la caractéristique interlocutive de l'échange langagier, les élèves peuvent dans un débat réglé profiter de toutes les postures énonciatives. Ils partent de la posture énonciative en "je », occupent la place " allocutive » pour interpeller les autres « $t u »$, " vous ", ils emploient le délocutif pour parler de l'autre ou d'eux - mêmes à la troisième personne, mais s'ils s'objectivent alors c'est pour mieux s'identifier subjectivement, puisque le il peut reprendre ce qu'on adit de lui à la troisième personne. Ils réagissent aux évaluations de l'autre et apprécient les effets perlocutoires du texte.

Cette virtuosité interlocutive très personnifiante est particulièrement remarquable dans un bref échange entre Julien et Johanna (I - 430 - 432)

Julien « Bah moi j'ai bien aimé le livre parce qu'il tue pas le lion» ...

Johanna : "Toi qu'aimes heu qu'aimes faire la bagarre maintenant tu es tu es ...non comme il aime faire la bagarre ça me fait un choc "...

Julien : « ouais mais je préfère la faire avec Guillaume qu'avec le lion ».

69 A la fin du débat, au moment où chacun exprime son évaluation profonde, Johanna comme les autres a sans doute été touchée par la formule de Julien. Lui le bagarreur avoue qu'il aime le message non violent de l'album. Probablement Julien a - $t$ - il opéré une prise de conscience grâce au texte et aux processus d'identification avec le personnage permis par la lecture et le débat. Chacun est témoin de son énonciation explicite et reçoit ce témoignage comme signe d'une transformation dans son identité : il se rapproche un peu plus du sujet qui a intégré l'interdit moral. La bonne coopération qui a prévalu a permis aux enfants de construire ensemble des interprétations validées par un retour fréquent au texte, ils ont mieux compris le sens du texte, ils se sont confortés dans leur participation à une communauté discursive lettrée. Ils se sentent valorisés, plus unis et plus proches, c'est sans doute la raison pour laquelle, à ce moment, Johanna peut s'autoriser à interpeller Julien sur sa réputation. Comment parvient - il sans contradiction à concilier son penchant pour l'échange des coups avec sa proclamation solennelle sur la 
supériorité du refus de tuer? Passant de l'allocutif «toi » au délocutif «il» elle prend à témoin le groupe tout entier en dramatisant l'effet perlocutoire "ça me fait un choc». L'atmosphère de bonne entente, permet aux enfants sécurisés de se dire leurs quatre vérités sans risque de se blesser. Julien ne s'y trompe pas : il a saisi la perche et répond avec humour. Il reprend la désignation délocutive du il qui le désignait comme non personne en je, pour se réinsérer pleinement subjectivement dans l'espace interlocutif Le nous des élèves dans cette communauté de débat réglé est inclusif : il englobe dans son extension le je et le tu, comme il a pu contenir l'effet toujours un peu dénonciateur du $i l$ délocutif. personne. Les didacticiens du Français comme Jean - Charles CHABANNE et Dominique BUCHETON $^{35}$ ou encore de la philosophie comme Michel TOZZI (op. cit.) ont insisté à juste titre sur l'importance de l'oral et de l'écrit réflexifs dans la classe. Reprenant l'héritage piagétien sur l'abstraction réfléchissante et vygostskyien sur la secondarité du langage, ils montrent que ces pratiques langagières en favorisant le retour sur la tâche, le texte, sa parole ou celle de l'autre nourrissent un accroissement de la conscience, elles sont simultanément facteur de cognition, de socialisation, et de subjectivation.

BENVENISTE, déjà en effet, inscrit le pouvoir réfléchissant du langage dans la vertu du pacte symbolique Par le symbolique, l'homme re - double, re - produit, re - présente, re crée la réalité (op.cit. p.25) cette faculté de redoubler le monde se fondant sur la communauté (co - venante) qui est le propre de l'humain et de son anthropogenèse : « entre la fonction sensori - motrice et la fonction représentative, il y a un seuil que l'humanité seule a franchi. (..) L'émergence de l'Homo dans la série animale peut avoir été favorisée par sa structure corporelle ou son organisation nerveuse; elle est due avant tout à sa faculté de représentation symbolique, source commune de la pensée, du langage et de la société. $»^{36}$

On peut plus précisément encore saisir cette propriété en considérant, à la suite de Francis JACQUES l'aspect interlocutif et relationnel du langage. Dans l'entreprise interlocutive chacun peut passer de la référenciation à la co - référenciation « ... on parle avec l'autre et non pas seulement à l'autre. C'est moi qui parle mais c'est nous qui disons. ${ }^{37}$ ”. Grâce aux jeux interlocutifs avec l'autre que permettent les textes et leurs genres, chacun 
peut se placer dans les différentes postures instantielles : je tu il, nous vous eux ... Par un parcours à travers le temps et la relation, par la médiation des trois instances et des genres interlocutifs, l'instance énonciative qui prend la parole peut advenir comme un soi réflexif et personnel. A l'issue du processus interlocutif, le sujet a conquis une autre épaisseur qu'au départ. Il a vécu la difficile identification intersubjective, il émerge d'une mise en jeu de soi (pronom réfléchi de toutes les personnes) à travers l'intersubjectivité et les trois instances du pronom personnel. La capacité tri - instancielle, c'est - à - dire l'aptitude de recouvrement des différentes places que propose le pronom, pour soi même comme pour l'autre, devient donc indispensable pour le développement de la personne et pour son identification personnelle. «Je dois être capable d'accueillir l'adresse ou l'interpellation d'autrui qui me dit tu, sous peine de ne pas être je. Si je suis celui auquel on se réfere en seconde personne ce tu alors c'est moi. (...) Pendant le même temps je dois être capable de me reconnaître comme l'objet d'un discours qui me concerne à la troisième personne; ce il alors c'est moi. Entre ces trois instances - je, tu, il - le lien est indissoluble. Le résultat est une unité structurelle d'une énorme complexité logique: la personne ${ }^{38}$. Ce processus d'identification passe par le tiers. Le tiers est une des positions essentielles du processus de personnification il ne saurait être rejeté dans le point aveugle de la non - personne ${ }^{39}$. Cette improbabilité selon Benveniste (que le «il» dise «je ») est démentie par le donné lui - même dans l'inventivité de certains textes, ou pratiques textuelles, au point d'autoriser malgré tout l'assomption du « je » par le «il » et de la personne par la non personne. Plus, elle devient condition de l'identification personnelle.

Le tiers personnel est la position la plus délicate de par la prédication (dis) qualificative de la délocution. On attribue au tiers des caractères et des prédicats qui le mettent aisément dans une posture d'objectivation sinon de réification. L'objet n'est pas sujet aussi l'identification subjective pourrait être irrémédiablement empêchée par cette contrainte. Cette condition prédicative de la subjectivité est paradoxale: comment confronter l'homme aux qualités qui lui sont imputables sans risquer de l'objectiver? On a vu avec Julien et Johanna comment la reprise délicate du prédicat de bagarreur devenait possible dans l'espace interlocutif pacifié du débat d'interprétation littéraire. Grâce à la commune identification au personnage de Yacouba, grâce à la connivence de la co construction des significations et des valeurs dans la communauté interprétative, Julien pouvait tranquillement accepter l'interpellation de Johanna, se reconnaittre dans la délocutive objectivation du 'il' bagarreur et avec humour, reprenant à son compte ce qu'on dit de lui, sans colère ni provocation victimaire, signifier toute la distance prise avec ce rôle. Une telle caractérisation objectivante n'est donc pas toujours obstacle à la subjectivation, bien au contraire. Dans un contexte qui associe de façon réglée le régime délocutif et le régime allocutif, on peut prendre en compte pour l'assumer ou le modifier ce qui a été dit de soi. On connait la propension mimétique de l'homme à se conformer à la qualification que lui propose l'autre, fut - elle dépréciative. L'autre est médiateur de moi à moi et comment imaginer un processus éducatif sans assumer la caractérisation évaluative de soi ou de ses rôles et attitudes? La caractérisation pourra aider d'autant mieux sur le plan éducatif au développement de la personne qu'elle est reliée à un processus, un agir communicationnel articulant dans le temps la variation des contextes et des postures allocutives et délocutives. À ce prix, elle semble être justement une condition d'émergence de la personne ${ }^{40}$. L'objectivation critique par l'autre, si elle est respectueuse peut être suivie d'une sui - référentiation ou retro - référenciation où sans cesser d'être un sujet, on accepte de s'objectiver, de s'examiner, se caractériser, et faire un retour rétrospectif sur soi. Ce chemin de réflexivité est nécessaire pour devenir une 
personne, un soi il faut se définir soi - même comme un autre ${ }^{41}$. C'est du moins ce que nous disent les philosophes personnalistes du langage comme JACQUES ou Paul RICEUR. L'identification subjective personnelle est une réidentication de soi réflexive qui passe par l'autre et par l'aveu de la ressemblance avec l'autre, elle permet alors non seulement de se dégager de l'objectivation excluante, mais aussi de l'identification confusionnelle non sue comme telle.

\section{Conclusion}

Il faut rappeler les défis énormes du débat à l'école. Ce genre scolaire peut devenir espace interlocutif de l'émergence de l'élève comme sujet réflexif et comme personne, à condition d'être pacifié, régulé et ritualisé par un adulte garant de ses règles, de manière à ce que chacun puisse se les approprier pour y trouver une place respectée et respectueuse des contraintes de la discussion rationnelle comme de l'autre. Car s'il est l'alternative à une relation pédagogique verticale et hétéronomique, il ne peut permettre l'accès à l'autonomie attendue pour l'élève qu'à condition de ne pas basculer dans une socialisation horizontale anomique : mimétismes, pressions et oppressions entre pairs, consensus mous et collusifs d'opinions. L'indifférenciation nivelante, la démagogie et la rhétorique sont une menace pour chacun dans les cultures de masse, pire encore pour les enfants chez qui elle empêche tout simplement l'accès à la pensée et à la réflexion.

77 A l'heure de l'individualisme négatif et de la rupture du lien social (R. CASTEL) où chacun se sent vulnérable et fragilisé dans l'estime de soi ou dans ses appartenances, l'enjeu de la définition d'une identité subjective et personnelle (singulière et solidaire) est majeur car aujourd'hui, cette identité est le bien le plus précieux que l'éducation puisse offrir à l'enfant.

78 Mais attention ce processus éducatif n'est pas déconnecté de l'instruction, bien au contraire, c'est dans et par les genres et textes du savoir, avec leurs spécificités disciplinaires, régulés comme des espaces interlocutifs respectueux que peuvent s'engager un processus d'inter - identification subjective réflexive. Chacun y gagne l'estime de soi dans le développement des compétences et des postures propres à l'affiliation discursive dans une communauté disciplinaire particulière.

79 Si l'approche anthropologique permet de mieux comprendre l'intérêt et l'impact d'une différenciation des textes et des personnes dans la classe, l'approche textuelle et interlocutive de la pragmatique personnaliste autorise à discerner les catégories et les critères qui pourront être mis en oeuvre dans le cadre d'une démarche interdidactique.

\section{NOTES}

1. L'anomie définie d'abord par DURKHEIM (Le Suicide) est la perte des repères communs traditionnels, prescriptions et proscriptions reconnus, elle engendre une violence interpersonnelle et intrapersonnelle qui peut aller jusqu'à l'autodestruction. L'anomie (a privatif 
et nomos règle, limite) n'est ni l'hétéronomie où l'on est soumis à la règle de l'Autre ni l'autonomie où le sujet consent librement à la règle.

2. Jean - Paul BERNIE, sous la dir. de ; (2001) ; Apprentissage, développement et significations; en hommage à Michel BROSSARD ; Presses Universitaires de Bordeaux.

3. Nicole BIAGIOLI. Voir Travaux de l'ERTE IDD, interdidactique des disciplines, IUFM de Nice.

4. MEN p. 49

5. voir TOZZI, M. ; (2001), L'éveil de la pensée réflexive à l'école primaire ; Hachette.

6. Voir BERNIE, J - P. ; idem.

7. Voir «Débat et formation» étude (2004 - 2006) conduite par Marie - Louise MARTINEZ au DIERF (Département interdisciplinaire d'études et recherches en formation) à l'IUFM de Nice.

8. GER: Groupe d'élaboration de ressources de l'IUFM de Montpellier, piloté par Michel TOZZI, avec le conseil scientifique de Dominique BUCHETON

9. Collectif d'études: Monsieur BARLET - ROUSSET (IEN), Mme Anne PIERA (conseillère pédagogique) et autres enseignants de la circonscription du Grau du Roi.

10. DEDIEU, Thierry, (1994) Yacouba, Seuil Jeunesse. Cet album de littérature jeunesse, malgré sa brièveté, par sa grande simplicité autant que par la complexité des processus humains mis à jour, par l'économie et la qualité des moyens est sans doute un modèle de ce genre littéraire. Voir, dans le $N^{\circ} 23$ de TREMA l'article de Marie - Louise MARTINEZ « Yakouba ou le passage d'un rite à l'autre » pp 85 - 99

11. Ecole élémentaire du Cailar (en Petite Camargue).

12. ROBLIN, S. (2003), La littérature au cycle 3; histoire de lire, histoire d'écrire, histoire de dire, Mémoire CAFIPEMF.

13. Nouveaux programmes de l'école élémentaire, 2002 ; " avoir compris et retenu que le sens d'une œuvre littéraire n'est pas immédiatement accessible, mais que le travail d'interprétation littéraire ne peut s'affranchir des contraintes du texte » CNDP, p. 194.

14. voir JACQUES, Francis. (1979), Dialogiques, recherches logiques sur le dialogue, PUF; (1982) Différence et subjectivité ; Aubier Montaigne ; (1985) L'espace logique de l'interlocution, PUF ; (2002) Ecrits anthropologiques, L'Harmattan ; (2002) De la textualité, ed. Jean Maisonneuve.

15. Ces catégories ont été déduites par l'analyse a priori de la signification, on en retrouve les critères dans la sémiologie de Charles Sanders PEIRCE, philosophe du langage américain. A la différence de SAUSSURE, PEIRCE considère le signe non seulement comme un interface signifiant / signifié, mais dans sa relation aux usagers, aux autres signes et surtout à la référence. Voir FRANCIS Jacques: "De la signifiance "; in Revue de Métaphysique et de morale, n 2, 1987. La vulgarisation de PEIRCE par le linguiste Lewis MORRIS (les dimensions sémantique, morphosyntaxique, pragmatique) a inspiré certains didacticiens de français. Le groupe EVA de didacticiens de français de l'INRP transpose ces notions linguistiques dans les travaux sur l'évaluation en français, de manière instrumentaliste mais très astucieuse. Il me semble, pour avoir pratiqué ces catégories tant avec des enseignants en formation qu'avec des élèves dans des pratiques de lecture / écriture en classe, qu'elles sont très utiles pour accompagner la lecture, l'écriture ou encore l'évaluation des textes. On peut, avec des élèves (en difficulté ou non), leur faire correspondre des questions catégorielles plus simples (de quoi parle le texte ? comment, au moyens de quels matériaux ? qui parle à qui et pour qui dans ce texte ?). Voir par exemple : Marie - Louise MARTINEZ (1991) « Le français en SES / EREA ou comment donner sens », in Les Cahiers de

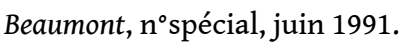

16. I et II renvoient respectivement à des corpus de transcription de séances orales, deux sont ici étudiés sur 4 séances de débat d'interprétation littéraire en classe (CM1 de Sophie ROBLIN à l'école élémentaire du Cailar). Chacun de ces groupes était constitué de 6 élèves sur 28 , la séance I a duré 29 minutes et la II 30 minutes. Les tours de parole ont été numérotés : 447 en I et 646 en II. 17. On peut entendre l'interprétation selon l'étymologie : un échange où chacun doit prêter dans l'interaction pour co - construire la signification. Il reçoit alors ses significations enrichies avec 
intérêts par celles de l'autre. Cette compréhension de l'interprétation, plus anthropologique qu'herméneutique, me semble davantage en adéquation avec la réalité rituelle de l'échange langagier.

18. GIRARD, René (1961) ; Mensonge romantique et vérité romanesque, Grasset ; (1994) SHAKESPEARE, les feux de l'envie, Grasset. Marie - Louise MARTINEZ (1998): "Pour une anthropologie relationnelle en littérature »; in Philosophie du langage esthétique et éducation; coord. par Patrick BERTHIER et Dany - Robert DUFOUR, L'Harmattan ; "Yacouba ou le passage d'un rite à l'autre ", Revue TREMA $\mathrm{N}^{\circ} 23$ in Approche anthropologique en éducation et en formation, 3e semestre 2004 ; Olivier MAUREL (2002) Essais sur le mimétisme; sept œuvres littéraires et un film revisités à la lumière de la théorie de René Girard ; L'Harmattan

19. Emile BENVENISTE (1966) «De la subjectivité dans le langage" in Problèmes de linguistique générale, Tome I, Gallimard. Il découvre l'ambiguïté du statut du tiers : « De sa fonction de forme non personnelle, la " troisième personne» tire cette aptitude à devenir aussi bien une forme de respect qui fait d'un être bien plus qu'une personne qu'une forme d'outrage qui peut le néantiser comme personne " p. 231 (souligné par nous).

20. KANT, Emmanuel $(1785 ; 1987)$ Fondements de la métaphysique des mœurs, Vrin.

21. LEVINAS, Emmanuel (1972) L'humanisme de l'autre Homme, Fata Morgana.

22. JACQUES, Francis (1985).L'espace logique de l'interlocution, PUF.

23. à certaines conditions méthodologiques, les sciences peuvent être un agir communicationnel de la personne, cela constitue pour moi la question épistémologique la plus importante.

24. BAKHTINE, Mikhaïl $(1979,1984)$, Esthétique de la création verbale, Moscou, (trad. du russe par T. TODOROV), Gallimard

25. La diégèse c'est la fable ou récit dans le langage d'ARISTOTE, premier théoricien du texte littéraire (La poétique).

26. MARTINEZ, Marie - Louise, (1991). «Le texte théâtral : à la croisée de deux dialogismes, l'émergence de la personne", in Le dialogue, MAURAND, Georges (sous la dir. de), Toulouse Le Mirail ; (1992), « Les pratiques textuelles théâtrales et l'enseignement du français », in Pratiques n ๑74, (coord. par Marie - Louise MARTINEZ et André PETITJEAN).

27. voir les travaux de l'ERTE IDD interdidactique des disciplines de l'IUFM de Nice dirigée par Nicole BIAGIOLI.

28. BENVENISTE, Émile, op. cit. p. 259.

29. « ... le je est un autre de Rimbaud fournit l'expression typique de ce qui est proprement l'aliénation mentale, où le moi est dépossédé de son identité constitutive ", BENVENISTE, op. cit. p. 230.

30. BENVENISTE, Émile, op. cit. p. 260.

31. BENVENISTE, Émile, op. cit. Tome I. p. 175.

32. "C'est un fait remarquable - mais qui pense à le remarquer tant il est familier ? - que parmi les signes d'une langue, de quelque type, époque ou région qu'elle soit jamais ne manquent les pronoms personnels. Une langue sans expression de la personne ne se conçoit pas. Il peut seulement arriver que, dans certaines langues, en certaines circonstances, ces " pronoms» soient délibérément omis; c'est le cas dans la plupart des sociétés d'Extrême - Orient, où une convention de politesse impose l'emploi de périphrases ou de formes spéciales entre certains groupes d'individus, pour remplacer les références personnelles directes. Mais ces usages ne font que souligner la valeur des formes évitées ", Émile BENVENISTE, op. cit. p. 261.

33. JACQUES, Francis, (1982), «Différence et subjectivité », Aubier Montaigne, p. 58.

34. Les notions de sujet et de personne sont des concepts clé de notre culture, définies par de nombreuses auteurs à la croisée de nombreux champs disciplinaires (théâtre, grammaire, théologie, philosophie, psychologie, sociologie, linguistique, psychanalyse, anthropologie, pédagogie, etc.) elles mériteraient de plus amples développements. Voir par ex : Marie - Louise MARTINEZ (1997) Vers La réduction de la violence à l'école; contribution à l'étude de quelques concepts pour une anthropologie relationnelle de la personne; thèse sous la direction du Professeur Francis JACQUES 
, Septentrion ; - (coordonnés par), (1999) «Sujet et personne », la Nouvelle Revue de l'AIS ; ${ }^{\circ} 4$ et $\mathrm{N}^{\circ}$ 5 ; (coordonné par), (2003) L'émergence de la personne ;éduquer, accompagner ; L'Harmattan.

35. CHABANNE, Jean - Charles et BUCHETON, Dominique, (2002), Parler et écrire pour penser, apprendre et se construire, L'écrit et l'oral réflexifs, PUF.

36. BENVENISTE, Émile, op. cit. p. 27.

37. JACQUES, Francis, idem.

38. JACQUES, Francis, op. cit. p. 51.

39. "Il n'y a pas d'identité personnelle sans un certain rapport au tiers absent ou lointain. On devra prendre en compte, pour l'intégrer, la troisième personne "; JACQUES, Francis, op. cit. p. 52.

40. "La seule façon légitime de parler d'autrui, c'est de le faire de telle manière qu'on pourrait en parler avec lui. La maxime qui suit, exigeante: " traite la personne d'autrui dans le discours que tu tiens sur elle, toujours en même temps comme un allocutaire possible. » Ainsi l'autre finira, sinon par se reconnaitre dans ce qui est dit de lui, du moins par reconnaître que c'est bien de lui qu'il s'agit. A considérer quelqu'un purement comme un il délocuté on lui rend quasi impossible la tâche du recouvrement identificatoire du moi. » JACQUES, Francis, (op. cit. p. 136).

41. RICCEUR, Paul (1990) Soi - même comme un autre, SEUIL.

\section{RÉSUMÉS}

Le débat et la discussion à l'école ouvrent la voie à un genre scolaire prometteur dont les effets sur l'élève: (transformation du rapport à l'école, au savoir, au langage, aux autres et à soi même) sont déjà largement démontrés. Cependant, si l'on veut répondre aux défis d'une véritable démocratisation de l'instruction comme de l'éducation, il est indispensable de faire du débat le lieu d'une double différenciation : celle des savoirs académiques ou disciplinaires selon leurs spécificités textuelles et discursives et celle des élèves comme sujets et personnes.

Prenant appui sur la transcription de séances de débat d'interprétation littéraire dans une classe primaire, on constate dans un premier temps comment ces élèves ont su saisir la spécificité différentielle du littéraire, on évoque ensuite comment ce débat est devenu l'espace interlocutif permettant l'iintersubjectivation des élèves comme personnes.

The debate and the discussion at the school develop like a new rich educational type having a positive effect on the pupils. Tranformation from pupil relationship with knowledge, langage, other one, or oneself have already proved their evidence ... Nevertheless, answering the stakes for a true democratisation requires the debate to be the space of a double differentiation.Differences between the various subject matters texts and discourses, and differences between the diversity of pupils subjective identity regarded as individual person.

Taking support on the transcription of meetings of literary debate of interpretation in a primary class we can see first how these pupil could tackle the very literary specific difference. Then we can see how such a debate session would become a real interlocutive space allowing pupil co identification as subject and individual person. 
INDEX

Mots-clés : approche textuelle, débat d'interprétation littéraire, différenciation, identification interlocutive

Keywords : differentiation, interlocutiv intersubjectiv identification, literature interpreting debate, textual approach

\section{AUTEUR}

\section{MARIE-LOUISE MARTINEZ}

Maître de conférences en sciences de l'éducation, IUFM Nice 